Deformation micromechanics: Deformation micromechanics studies were carried out using simultaneous Raman spectroscopy and either tensile or 4-point bending tests. For tensile deformation, an Instron 1122 testing machine was used simultaneously with the remote Raman Renishaw system 100. The 4-point bending tests were carried out according to the ASTM standard using a rig attached to the stage of the optical microscope of a Renishaw system 1000. The $632.8 \mathrm{~nm}$ red line of He-Ne laser was used in both the Renishaw 100 and 1000 systems. In all cases, the strains in coatings were measured independently using Series EA strain gauges with $6 \mathrm{~mm}$ gauge length and with a nominal resistance value of $120 \Omega$ (Measurements Group Inc.).

Final version: June 10, 2004

[1] P. Colomban, J. Corset, J. Raman Spectroscopy 1999, 30 , 863.

[2] B. Dietrich, K. F. Dombrowski, J. Raman Spectroscopy 1999, 30, 893.

[3] H. Miyagawa, D. Kamiya, C. Sato, et al., J. Mater. Sci. 1999, 34, 105.

[4] B. D. Batchelder D. N., J. Polymer Sci., Polymer Physics Ed. 1979, 17, 569.

[5] A. J. Campbell, C. K. L. Davies, D. N. Batchelder, Macromol. Chem. Phys. 1998, 199, 109.

[6] J. L. Stanford, R. J. Day, X. Hu, et al., Progr. Organic Coat. 1992, 20, 425

[7] X. Hu, J. L. Stanford, R. J. Day, et al., Macromol. 1992, 25,684 .

[8] X. Hu, R. J. Day, J. L. Stanford, et al., J. Mater. Sci. 1992 $27,5958$.

[9] X. Hu, J. L. Stanford, R. J. Young, Polymer 1994, 35, 80.

[10] C. A. Cooper, R. J. Young, J. Raman Spectroscopy 1999, 30, 929 .

[11] C. A. Cooper, R. J. Young, M. Halsall, Composites Part A 2001, 32, 401.

[12] Z. Jia, Z. Wang, C. Xu, et al., Mater. Sci. Eng. A 1999, 271, 395.

[13] J. R. Wood, Q. Zhao, M. D. Frogley, et al., Phys. Rev. B 2000, 62, 7571.

[14] J. R. Wood, Q. Zhao, H. D. Wagner, Composites Part A 2001, 32, 391.

[15] Q. Zhao, M. D. Frogley, H. D. Wagner, Composites Sci. Technol. 2002, 62, 147.

[16] M. S. Dresselhaus, G. Dresselhaus, A. Jorio, et al., Carbon 2002, 40, 2043.

[17] M. D. Frogley, Q. Zhao, H. D. Wagner, Phys. Rev. B 2002, 65, 113413.

[18] G. S. Duesberg, I. Loa, M. Burghard, et al., Phys. Rev. Lett. 2000, 85, 5436.

\section{Processing of Transparent Conducting Coatings Made With Redispersible Crystalline Nanoparticles}

\author{
By Joerg Puetz, * Naji Al-Dahoudi, and \\ Michel A. Aegerter
}

Recent developments in display and smart window technology depend fundamentally on transparent electrodes, both for electrical control and antistatic purposes. The required combination of an electrical conductivity with a high transmission in the visible range is found in a class of materials that is referred to as "transparent conducting oxides" (TCO) with ITO $\left(\mathrm{In}_{2} \mathrm{O}_{3}: \mathrm{Sn}\right)$, FTO $\left(\mathrm{SnO}_{2}: \mathrm{F}\right)$, ATO $\left(\mathrm{SnO}_{2}: \mathrm{Sb}\right)$ and (AZO) $\mathrm{ZnO}: \mathrm{Al}$ being the most frequently used. ${ }^{[1]}$ All these oxides are n-type doped wide band gap (> $3 \mathrm{eV}$ ) semiconductors in which doping is accomplished by exchange of oxygen (oxygen vacancies, $\mathrm{F}^{-}$) or replacement of a metal ion with an ion of higher oxidation state (e.g. $\mathrm{Sn}^{\mathrm{IV}}$ in $\mathrm{In}_{2} \mathrm{O}_{3}$ ).

TCOs are always applied as thin films with a thickness much below $1 \mu \mathrm{m}$ to keep the transmission high. The method of choice for display applications is magnetron sputtering as it gives the lowest resistivity with the best optical quality for ITO and AZO, but for architectural applications chemical vapor deposition (CVD) or spray pyrolysis of FTO are favored because of the much lower deposition cost. ${ }^{[2]}$. All these processes require elevated temperatures of at least $200^{\circ} \mathrm{C}$ (sputtering) to $500{ }^{\circ} \mathrm{C}$ (spray pyrolysis), so that coatings can only be deposited on glass substrates. The increasing demand for portable consumer electronics, however, is calling for a change for the lighter plastic films as substrates, but with a similar or only slightly decreased electrical conductivity.

There are currently two competing approaches aiming at the deposition of TCO coatings on plastics. One is based on the modification of magnetron sputtering for a semi-continuous deposition on plastic webs, ${ }^{[3]}$ but the technical expenses still make the coatings too expensive for most applications and the electrical properties are often insufficient. Alternatively to these vacuum deposition techniques, wet coating techniques have already proven their suitably for web coating in industrial processes like for photographic films or the surface modification of papers. ${ }^{[4]}$ The sol-gel process that is wellknown for oxide coatings, however, cannot be used for plastics as high temperatures are required to obtain conducting films. ${ }^{[5]}$

[*] Dr. J. Puetz, Dr. N. Al-Dahoudi, Prof. M. A. Aegerter Leibniz-Institut für Neue Materialien - INM

Saarbruecken, Germany

E-mail:puetz@inm-gmbh.de 
a)

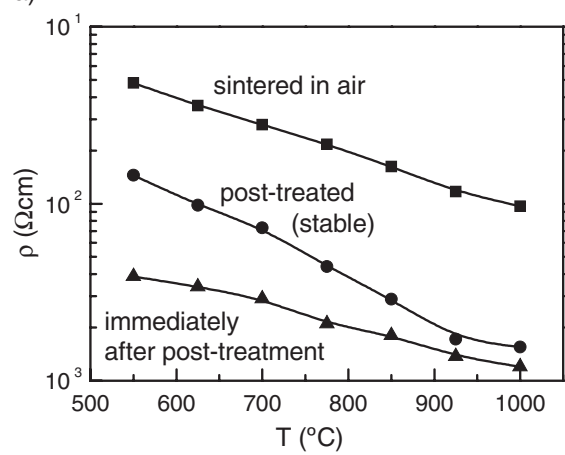

c)

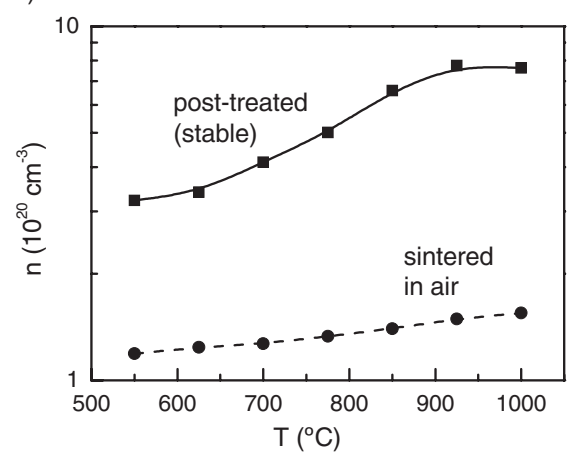

b)

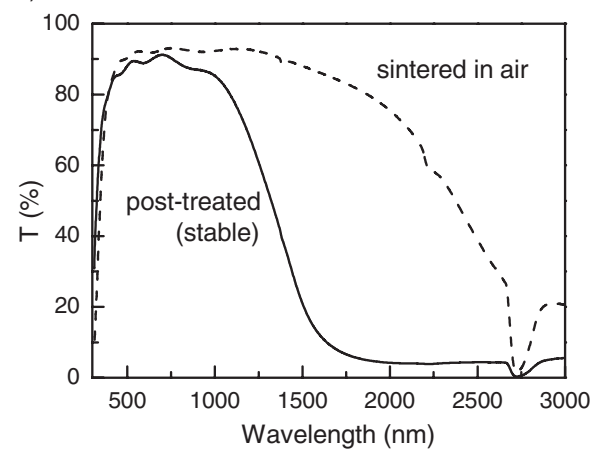

d)

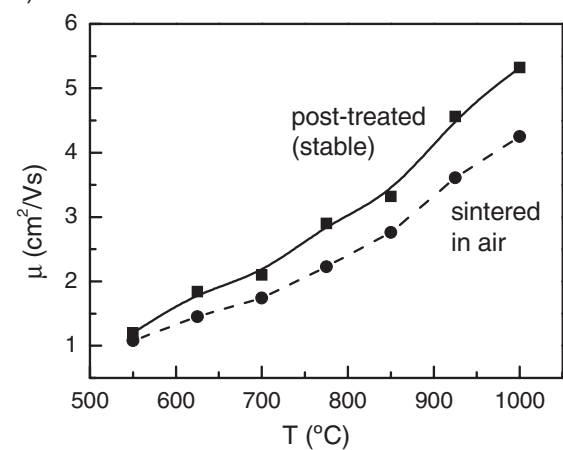

Fig. 1. Electrical and optical properties of ITO nanoparticle coatings (t $570 \mathrm{~nm})$ on borosilicate glass after sintering in air at $550{ }^{\circ} \mathrm{C}$ and after post-treatment under forming gas at $350{ }^{\circ} \mathrm{C}(30 \mathrm{~min})$ and subsequent equilibration in air, respectively.

For a coating of plastic substrates it is therefore necessary to break new ground in TCO deposition by developing materials that can be processed completely at low temperatures.

The use of crystalline TCO nanoparticles ${ }^{[6]}$ is one of the most promising approaches for a wet chemical processing of such coatings. The fundamental advantage of this processing is the separation of the heat treatment that is required both for the crystallization of the material and the formation of charge carriers under reducing conditions, from the process of film formation. This offers new ways for the deposition and curing of functional films in general by modifying and tailoring the surface of the particles chemically. ${ }^{[7]}$ Another advantage of nanoparticles is found in the fact that, for particles smaller than about $30 \mathrm{~nm}$, optical light scattering can be reduced to a minimum which guarantees a high transmission in the visible range. For the electrical properties, however, the particle size should be as large as possible to keep the grain boundary scattering low, so that in practice a compromise between the optical and electrical properties favors a particle size of 20 to $30 \mathrm{~nm}$.

For transparent conducting coatings currently two classes of materials are employed in the nanoparticle approach. Socalled "organic metals", like polyaniline and polyethylene-dioxythiophene (PEDT), ${ }^{[8,9]}$ have attracted considerable interest and, meanwhile, are commercially available in ready-to-use compositions. Such materials can be prepared with a particle size below $10 \mathrm{~nm}$ and can easily be incorporated in different matrices with a final resistivity of $1 \times 10^{-2} \Omega \mathrm{cm}$. However, these materials suffer from a low UV stability and an increased absorption in the visible, which limits their application.

Pure inorganic TCOs, in contrast, show a much higher UV and chemical resistance with a higher transmission in the visible but with similar electrical properties. Meanwhile, redispersible nanoparticles of ITO, which shows the best electrical properties among the TCOs, can be produced reproducibly on an industrial scale and various products are on the market. The present paper describes recent developments in the employment of such ITO nanoparticles for the preparation of transparent conducting coatings and discusses capabilities and limits of this approach.

Experimental: Redispersible nanoparticles of ITO $\left(\mathrm{In}_{2} \mathrm{O}_{3}: \mathrm{Sn}\right)$ with a doping level of $8 \mathrm{~mol} \%$ and a particle size of 20 to $30 \mathrm{~nm}$ were prepared wet-chemically by a controlled growth process that has already been described in detail previously. ${ }^{[6]}$ In order to obtain the desired tin doped $\operatorname{In}_{2} \mathrm{O}_{3}$ phase and to increase the number of charge carriers, the powder can be reduced in a forming gas atmosphere at temperatures up to $350^{\circ} \mathrm{C}$ without affecting the redispersibility. ${ }^{[10]}$ For a further processing this powder is de-agglomerated mechanically with the help of a mixture of ethylene glycol and diethylene glycol monobutyl ether (50/50 by volume) and a carbonic acid for surface modification to form a paste with a solid content of approximately $70 \mathrm{wt}-\%$ ITO. The resulting paste is stable for several months without any evidence of agglomeration or precipitation.

Coating sols are prepared by diluting the paste with ethanol and centrifugation of residual agglomerates to give a final ITO content of $25 \mathrm{wt}-\%{ }^{[6,10]}$ For the low temperature processing, pre-hydrolyzed 3-methacryloxypropy-trimethoxsilane (MPTS) is added in an amount of approx. $6 \mathrm{wt}-\%$ as a binding agent together with a UV photostarter (Irgacure 184).

Transparent conducting films with a thickness ranging between 0.5 and $1.0 \mu \mathrm{m}$ were deposited on $10 \times 10 \mathrm{~cm}^{2}$ glass (borosilicate, fused silica) or plastic substrates (PMMA, PC, polyesters) using wet deposition techniques such as spin or dip coating. The wet films then were either sintered at high temperatures in air $\left(500\right.$ to $1000^{\circ} \mathrm{C}$ ) or cured at low temperatures by UV irradiation (BELTRON, typically $105 \mathrm{~mW} / \mathrm{cm}^{2}$, $110 \mathrm{~s})$ combined with a subsequent heat treatment at $130^{\circ} \mathrm{C}$ during several hours. An additional annealing under reducing (forming gas, $\mathrm{N}_{2} / 8$ vol- $\left.\% \mathrm{H}_{2}\right)$ or inert atmosphere $\left(\mathrm{N}_{2}\right.$, 

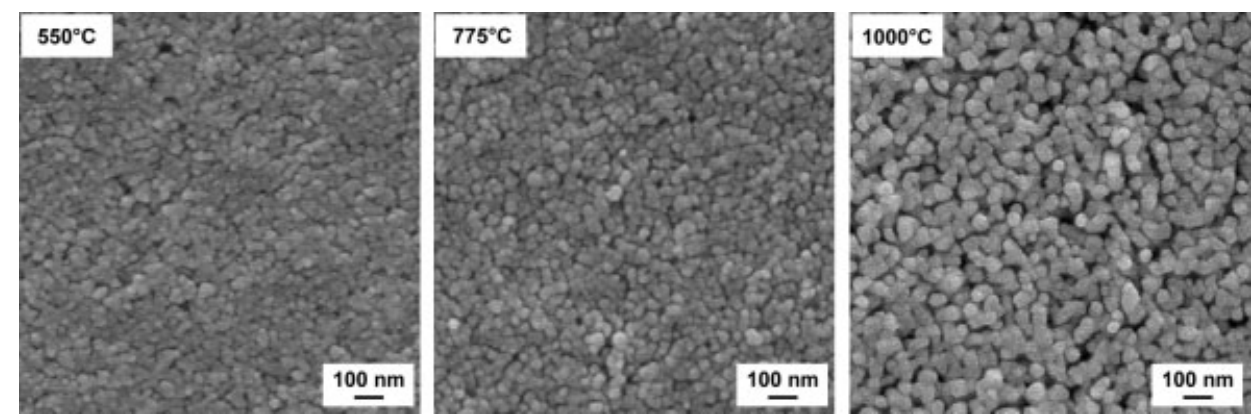

Fig. 2. SEM morphology of pure ITO nanoparticle films after sintering at temperatures of $550{ }^{\circ} \mathrm{C}, 775^{\circ} \mathrm{C}$ and $1000{ }^{\circ} \mathrm{C}$, respectively.

Ar) at $130{ }^{\circ} \mathrm{C}$ during $2 \mathrm{~h}$ further reduces the sheet resistance of the coatings.

The structural properties of the coatings were investigated using profilometry for the film thickness (P10, Tencor) and both scanning electron microscopy (SEM - JSM 6400F, JEOL; $10 \mathrm{kV}$ ) and cross-sectional high resolution transmission electron microscopy (HRTEM - CM200 FEG, Philips; $200 \mathrm{keV}$ ) for the surface and bulk morphology, respectively. Surface images of ITO patterns on larger scale were taken by a white light interferometer (WLI - Newview 5000, Zygo). The electrical properties were measured using a four-point probe and a combined van der Pauw/Hall measurement (MMR Technologies). Optical transmission spectra were collected for coatings on fused silica substrates against air reference (CARY 5E, Varian). The mechanical properties were determined according to standard test methods in terms of the adhesion (tape test - DIN 58196-K2; cross cut test - ASTM 3359 / DIN 53151), the abrasion resistance (cotton cloth and hard rubber DIN 58196-H25/G10; taber test - DIN 52347/CS10F/ $5.4 \mathrm{~N}$ ) and the hardness (pencil test - ASTM 3363-92a).

Results and Discussion

High temperature processing: Transparent conducting ITO coatings can be prepared readily from dispersions of surface modified ITO nanoparticles by wet deposition followed by a conventional thermal treatment as in sol-gel processing. Due to the higher solid content and the lower shrinkage of the crystalline nanoparticles the resulting coatings have a significantly higher thickness of approx. $570 \mathrm{~nm}$ with a high transmission in the visible range (Fig. 1b, dashed line). But the corresponding electrical properties (Fig. 1a) with a resistivity of $5 \times 10^{-2} \Omega \mathrm{cm}$ (corresponding to a sheet resistance of $1.2 \mathrm{k} \Omega_{\square}$ ) after sintering at $550{ }^{\circ} \mathrm{C}$ in air are not yet satisfying and at lower processing temperatures the sheet resistance is even in the order of several $\mathrm{M} \Omega$.

An improvement of the conductivity can be achieved by a post-treatment under reducing conditions with a minimum at $4 \times 10^{-3} \Omega \mathrm{cm}$ after annealing in forming gas $\left(350^{\circ} \mathrm{C}, 30 \mathrm{~min}\right)$. This value is, however, not stable under ambient conditions in air and finally reaches $1.5 \times 10^{-2} \Omega \mathrm{cm}$ after several days, which nevertheless is lower than the original value. The mechanism behind this reversible process is not yet fully understood, but it is certainly connected to the formation of oxy- gen vacancies. Though the ITO nanoparticles were already treated in forming gas atmosphere during the synthesis, it is likely that a fraction of the oxygen vacancies especially at the particle surface gets lost during the wet processing and the high temperature treatment in air. These oxygen vacancies can be recovered in a post-treatment step under inert or reducing atmosphere but under exposure to oxygen the number of charge carriers is decreased again at the particle surface resulting in an increased resistivity. As the latter process usually takes place at room temperature it affects the number of charge carriers only superficially and hence the observed resistivity is still lower than after annealing in air. This model is supported by Hall measurements (Figs. 1c and 1d) which clearly show that the decreased resistivity after forming gas treatment is largely due to the higher density of charge carriers rather than to their mobility.

With increasing sintering temperature, the resistivity is lowered by a factor 4 to a value of $1.2 \times 10^{-2} \Omega \mathrm{cm}$ at $1000^{\circ} \mathrm{C}$ (Fig. 1a) coming along with a particle growth from 25 to $33 \mathrm{~nm} .{ }^{[10]}$ The post-treatment again leads to a significant decrease in the resistivity, but here the value after equilibration in air is much closer to the minimum value measured immediately after post-treatment. This becomes plausible if we consider that the interacting surface area is smaller for the larger, sintered particles and hence the influence on the total number of charge carriers is lower. The Hall measurements reveal that in fact the charge carrier density is responsible for this approximation of the values. But also the mobility of the
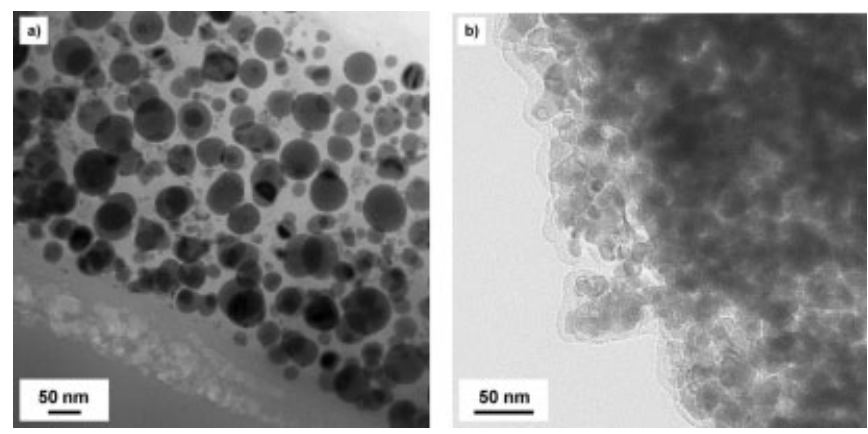

Fig. 3. HRTEM images of ITO/MPTS composites after a) heat treated at $130{ }^{\circ} \mathrm{C}$ and $b$ ) UV curing. 


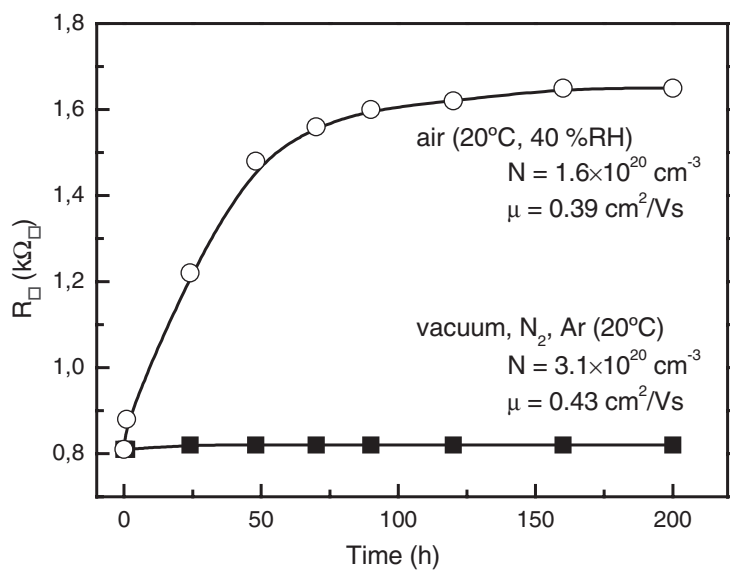

Fig. 4. Evolution of the sheet resistance of post-treated ITO/MPTS nanocomposite coatings during storage in air or under inert conditions (vacuum, $N_{2}, A r$ ), respectively. The thickness of the coatings is approx. $570 \mathrm{~nm}$.

charge carriers is slightly higher which can be interpreted in terms of larger crystallites and better interconnections between them.

As a result of the close relationship between the electrical and optical properties, the electronic changes during the post-treatment also show in the optical spectra (Fig. 1b). The forming gas treatment leads to a significant decrease of the transmission above $800 \mathrm{~nm}$, which can only to a certain extent be explained by the increased density of charge carriers and the corresponding shift of the plasma edge. Instead, an additional absorption of the ITO in the near IR (800 nm to $2.5 \mu \mathrm{m}$ ) is clearly seen in the absorption spectra ${ }^{[10]}$ possibly connected to defects in the material due to the oxygen deficiency.

The particle growth and the coalescence with increasing sintering temperature can be clearly observed in the SEM images as shown in Figure 2. The coatings still exhibit a granular structure with a high porosity explaining the significantly higher resistivity of these films compared to sputtered films (typically $1 \times 10^{-4} \Omega \mathrm{cm}$ ). The reason for this porosity is basically found in the spherical shape of the particles but also the surface modification and the organic solvents certainly impede a closer approach of the particles during film drying.

The loose structure with only isolated sintering necks already suggest poor mechanical properties of such coatings and in fact they can be wiped off even after sintering at $1000^{\circ} \mathrm{C}$ (cloth test: class 2, tape test: passed $^{[10]}$ ). A paramount task consequently is aiming on an improvement of the mechanical properties and the density to decrease the resistivity.

Low temperature UV processing: In order to improve the cohesion between the ITO nanoparticles, a chemical binder based on an organically modified silane was added to the ITO coating dispersion. Such organically modified silanes offer highest flexibility for film processing and curing due to their hybridic nature. ${ }^{[11]}$ By a pre-hydrolysis an inorganic silicate-like network can be formed with a high mechanical strength and the possibility to bind to the inorganic ITO surface chemically. The choice of an organic side chain with special chemical functionalities, however, makes new curing methods possible as it has been realized using a double bond containing 3-methacryloxypropyl-trimethoxsilane (MPTS). In the presence of a photo initiator such coating systems now can be UV cured by radical polymerization at low temperature.

The role of the UV illumination for the morphology in the curing process of such ITO/MPTS nanocomposites has been investigated using electron microscopy (Fig. 3). With a moderate heating at $130^{\circ} \mathrm{C}$ without UV light, the resulting coatings exhibit a dense surface structure but the cross-sectional HRTEM image (Fig. 3a) shows isolated ITO particles embedded in an amorphous matrix. This morphology very much resembles an emulsion like structure with agglomerates of the smaller ITO nanoparticles. Accordingly, the electrical conductivity of these samples is poor with a sheet resistance of several $\mathrm{M} \Omega$.

In contrast, the UV cured samples (approx. $10 \mathrm{~J} / \mathrm{cm}^{2}$ ) shown in Figure 3b, exhibit a granular but cohesive structure with the ITO nanoparticles in direct contact and in their original particle size, i.e., without agglomeration. This structure allows a continuous conduction which shows in the much lower sheet resistance of the UV cured sample of only $8 \mathrm{k} \Omega$. These results indicate that it is rather the polymerization of the double bond that is beneficial for the conductivity than the contribution of the pre-hydrolysed MPTS binder itself.

A sheet resistivity of $8 \mathrm{k} \Omega$ obtained after UV curing in air corresponds to a resistivity of $0.4 \Omega \mathrm{cm}$ for a $570 \mathrm{~nm}$ thick coating which is still at least three orders of magnitude higher than the best values obtained for sputtered coatings. But like in the case of the pure ITO films, a further decrease again can be achieved by a reducing post-treatment at low temperatures (up to $130^{\circ} \mathrm{C}$ ). Figure 4 shows the temporal evolution of the sheet resistance of such ITO/MPTS nanocomposite coatings after a forming gas treatment. While the sheet resistance a)

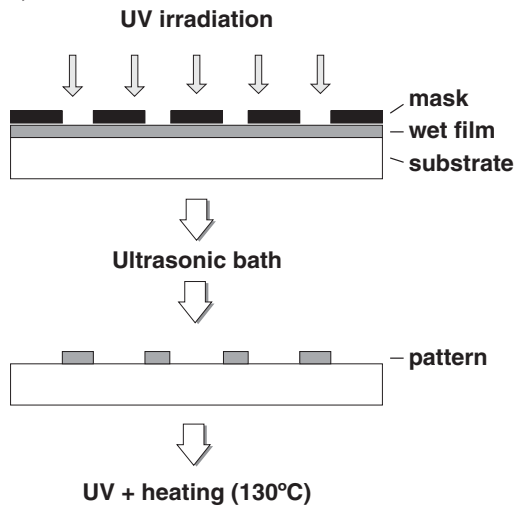

b)

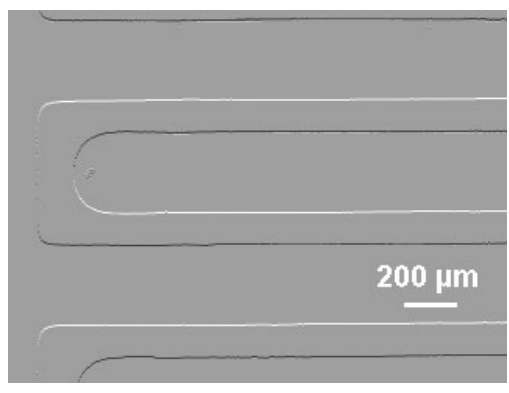

Fig. 5. Mask illumination procedure for wet films (a) and obtained ITO pattern on a PC substrate (b). 
is stable under inert storing conditions (vacuum, $\mathrm{N}_{2}, \mathrm{Ar}$ ), it slowly increases by a factor of 2 reaching a constant value of $1.6 \mathrm{k} \Omega_{\square}$ after more than $150 \mathrm{~h}$. It is most probably the same mechanism of oxygen adsorption and the loss of charge carriers that is responsible for this increase in sheet resistance. Compared to the pure ITO films, however, the process is rather slow as the density is higher and thus the diffusion of oxygen should be hindered. A closer investigation is confirming this assumption revealing that the increase is mainly caused by a drop in the charge carrier density from 3.1 to $1.6 \times 10^{20} \mathrm{~cm}^{-3}$ whereas the mobility remains almost constant (0.39 and $0.43 \mathrm{~cm}^{2} / \mathrm{Vs}$, resp.).

The optical properties of the UV cured composite coatings in the visible range are similar to those of pure ITO (Fig. 1b) with a transmission larger than $85 \%$ in the visible. Despite of the low content of the binder, the mechanical properties of the respective coatings could be improved significantly compared to the pure inorganic material. The coatings pass both the tape and the cross cut test, certifying a good adhesion even on plastic substrates. A testing of the abrasion resistance revealed no scratches after 10 rubbing cycles or after 25 rubbing cycles with the milder cotton cloth (both class 1 ). The results of the taber test with a haze of $15 \%$ after 10 cycles, however, indicate that the coatings are still rather soft, which is confirmed by a hardness of $1 \mathrm{H}$ determined by the pencil test. Superior mechanical properties could of course be obtained by an increased binder content, but this inevitably increases the resistivity of the coatings, so that in practice always a compromise has to be found between the electrical and mechanical properties of such TCO nanocomposite coatings.

UV patterning: The UV curing of ITO/MPTS nanocomposites cannot only be used to cure coatings but it can also be of advantage in the production of transparent conducting patterns, for example, for conductor lines or sensors. A common patterning method is found in the mask illumination process (Fig. 5a) in which the ITO film is covered with an impervious mask and is then exposed to UV light. In the exposed areas, the MPTS polymerizes and becomes insoluble in most solvents while the wet unexposed part of the film can easily be removed with ethanol in an ultrasonic bath. The remaining pattern is then again UV cured and heated to $130^{\circ} \mathrm{C}$ to complete the polymerization and evaporate the solvents. Typical line widths that can be realized with this method are of the order of several tens of microns (Fig. 5b). Finer structures can be obtained by a direct laser writing process (e.g. at $350 \mathrm{~nm}$ ) where the line width is basically determined by the diameter and the shape of the laser beam. ${ }^{[12]}$

Conclusions: Redispersible nanoparticles of transparent conducting ITO can be prepared reproducibly and in adequate amounts for industrial applications by a wet chemical processing. Dispersions obtained from such powders are characterized by a marked long-term stability and can be used in any kind of wet coating or printing technique to obtain transparent conducting coatings. Pure coatings of ITO nanoparticles, however, exhibit insufficient mechanical prop- erties even after sintering at $1000^{\circ} \mathrm{C}$ as the cohesion between the particles is still poor. It is therefore necessary to add a chemical binder like MPTS which both increases the load capacity and offers possibilities of a low-temperature processing. This way, transparent conducting coatings of an ITO nanocomposite with a thickness of up to $1 \mu \mathrm{m}$ and a sheet resistance of 1 to $2 \mathrm{k} \Omega_{\square}$ can be obtained on plastic substrates by a fast and flexible UV curing process, that can also be utilized for patterning. Such ITO nanocomposites therefore have to be considered a serious alternative to vacuum deposition or wet deposited organic metals in the deposition of transparent conducting coatings on plastics.

[1] H. L. Hartnagel, A. L. Dawar, A. K. Jain, C. Jagadish, Semiconducting Transparent Thin Films, Institute of Physics Publishing, Bristol, 1995.

[2] H. J. Gläser, Large area glass coating, Von Ardenne Anlagentechnik, Dresden, 2000.

[3] F. Milde, J. Brückner, C. Deus, M. Kammer, H. Neumann in: Proc. 4th Int. Conf. on Coatings on Glass, ICCG, 2002, p. 419-424.

[4] S. F. Kistler, P. M. Schweizer (Eds.), Liquid Film Coating, Chapman \& Hall, London, 1997, Ch. 11.

[5] J. Puetz, M. A. Aegerter, in: Handbook on Sol-Gel Technologies for Glass Producers and Users, Kluwer Academic Publishers (Norwell) (2004). accepted.

[6] R. Nonninger, C. Goebbert, H. Schmidt, R. Drumm, S. Sepeur, US Patent 6.533.966, 1999.

[7] D. Burgard, C. Kropf, R. Nass, H. Schmidt, MRS Symp. Proc. 1994, Vol. 346, p. 101-107.

[8] B. Wessling, Synth. Metals 2003, 153, 265.

[9] B. L. Groenendaal, F. Jonas, D. Freitag, H. Pielartzik, J. R. Reynolds, Adv. Mater. 2000, 12, 481.

[10] N. Al-Dahoudi, PhD Thesis, INM and University of Saarland, Shaker Verlag, Aachen, 2003.

[11] H. K. Schmidt, Macromol. Symp. 1996, 101, p. 333-342.

[12] M. A. Aegerter, N. Al-Dahoudi, A. Solieman, H. Kavak, P. Oliveira in: Proc. 7th ICFPAM, June 10-15, 2003, Bucharest, Romania. 